\title{
Galvanometer scanner modeling for Selective Laser Melting deflection system simulation
}

\author{
Fetra Rasoanarivo $^{1}$, Pedro Rodriguez-Ayerbe ${ }^{1}$ and Didier Dumur ${ }^{1}$
}

\begin{abstract}
Modeling of the actuating element of the three axis galvanometer based actuator for Selective Laser Melting (SLM) additive manufacturing (AM) process is addressed in this paper. Dynamic behavior of the single axis galvanometer motor actuator is studied with physical considerations that improve basic linear and simplified existing models and extend the frequency domain validation of the proposed model. Optimal feedback and feedforward-type control structure synthesis is derived through a black-box identification of the actual industrial system for the models validation purpose. Responses of the developed models are compared to experimental data. Modeling errors coming from both the actuator behavior (angular position values) and the marking process qualities (marking and focusing planes geometrical parameters) are found to be sufficiently small to allow the developed simulator to be used as an entry point for future investigations on the the single axis motor and the complete three axis actuator system.
\end{abstract}

\section{INTRODUCTION}

Additive manufacturing (AM) has become over the past decade a promising approach in rapid prototyping and is being exploited as an alternative for serial manufacturing of mechanical parts. AM technology allows complex mechanical parts design and presents interesting ecological indicators [2] as resource savings, eco-design optimization and toxic chemicals reduction compared to conventional manufacturing methods. AM techniques have applications in various fields [6] such as automotive, aerospace and medical industries. The most widespread AM technique for metal part manufacturing is the layer-by-layer fabrication method from selective melting of a metal powder bed. This technique knows continuous improvement due to the strong interdependence between the final part properties and the process parameters [9], [13]. Two main alternatives are used in current industrial machines as power sources for the selective melting of the powder bed [9], [13]: an electron beam and a high power laser which yield respectively the EBM (Electron Beam Melting) and the SLM (Selective Laser Melting) processes.

The actuator system for the laser power source in the SLM technique is investigated in this paper, being an important issue in the overall process performance. In the SLM process, the three main parameters that have the most important impact on the final part properties are [2], [9]: the single layer thickness on the metal powder bed, the scanning speed of the actuator and the laser beam diameter. The last two parameters compel an adequate understanding of the SLM

\footnotetext{
${ }^{1}$ Laboratoire des Signaux et Systèmes (L2S UMR8506), CentraleSupélec - CNRS - Univ. Paris-Sud, Université Paris-Saclay, Control Department, Plateau du Moulon, 91190 Gif-sur-Yvette, France

e-mail: fetra.rasoanarivo@centralesupelec.fr
}

actuator system behavior designated as the three axis scan head.

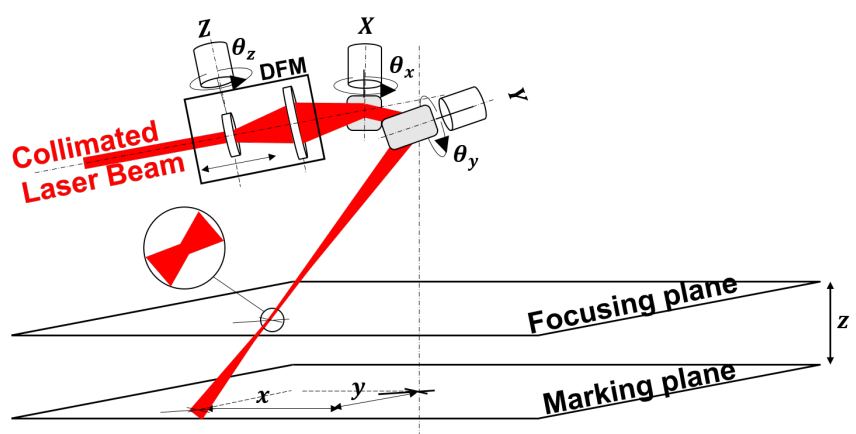

Fig. 1. The three-axis laser marking actuator configuration

The three-axis industrial marking system for SLM consists of two parts as depicted in Fig. 1. The first part is the laser deflection element which consists of the galvanometers $X$ and $Y$ with mounted mirrors, it allows to control the $(x, y)$ coordinate of the laser marking point in the marking plane. The second part of the system is the Dynamic Focus Module (DFM) element. The main purpose of this module is to control the $z$ height of the laser beam waist focusing plane, hence the laser beam diameter in the marking plane. The DFM consists of two elements [17]: a mobile expander lens to achieve the desired laser focusing control and a fixed objective lens. The translation movement of the expander lens is either achieved by linear dynamic motors as in [17] or by a rotary-to-linear movement conversion mechanism conducted by a $Z$ axis galvanometer as in Fig. 1, which is the case for the system investigated in the present work for experimental validation purpose.

The galvanometer motor has many application fields as: optical scanning for microscopy [12], precise laser drilling [3], [10], laser marking [5], laser trimming [11]. A first level of modeling considers the galvanometer motor as a rigid body, either in its simplest form as a second-order transfer function [5] or as a third order transfer function as a Direct Current (DC) motor with the two mechanical and electrical time constants [3], [12]. A second level of modeling exists which takes into account the torsion elasticity phenomenon at the mechanical link between the motor and its driven load [10], [11]. The proposed galvanometer modeling in this paper depicts a new way to approach the flexible torsion modes. A consideration of friction torques is also introduced to deal with low frequencies disparities that conventional models present. 
The paper is structured as follows. Section II focuses on the galvanometer modeling, including flexible modes and motor friction. Section III is dedicated to model validation, with a comparison with experimental data. Section IV finally proposes some conclusions and perspectives.

\section{GALVANOMETER DYNAMiCS MODELING}

A typical closed-loop configuration of a galvanometer scanner-based actuator as presented in [1] consists mainly of two parts: the motor actuator and the position controller. The modeling of the motor actuator based on physical considerations is addressed below.

\section{A. Motor multi-load representation}

Let us consider the galvanometer as a DC motor driving a load that can be divided into $n$ small load parts successively connected by mechanical links with elasticity coefficients $k_{j}$ for $j=1$ to $n$ as in Fig. 2 where $U$ is the voltage applied

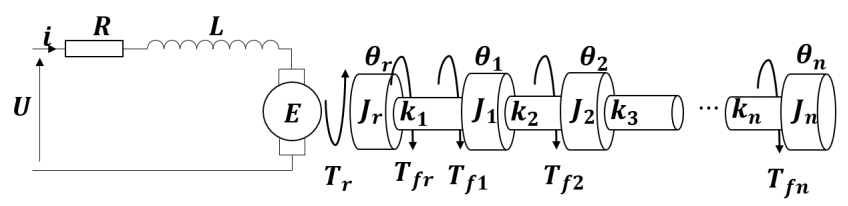

Fig. 2. The motor multi-load partition representation

to the motor coil, $i$ is the current flowing through the motor coil, $R$ and $L$ are the resistance and the inductance of the motor coil, $E$ is the counter electromotive force, $T_{r}$ is the motor torque, $\theta_{r}, \theta_{1}$ to $\theta_{n}$ are the rotor and the small loads angular positions, $J_{r}, J_{1}$ to $J_{n}$ are the inertia moment of the rotor and the small loads, $T_{f r}, T_{f 1}$ to $T_{f n}$ are the friction torques at the rotor and the small loads, $k_{1}$ is the elasticity coefficient of the mechanical link between the rotor and the first small load and $k_{2}$ to $k_{n}$ are the elasticity coefficients of the mechanical links between the remaining small loads. Equations (1) are derived from the Fig. 2 representation.

$$
\left\{\begin{array}{l}
U=R i+L \frac{d i}{d t}+E \\
J_{r} \ddot{\theta}_{r}=T_{r}-T_{f r}-k_{1}\left(\theta_{r}-\theta_{1}\right) \\
J_{1} \ddot{\theta}_{1}=k_{1}\left(\theta_{r}-\theta_{1}\right)-T_{f 1}-k_{2}\left(\theta_{1}-\theta_{2}\right) \\
J_{2} \ddot{\theta}_{2}=k_{2}\left(\theta_{1}-\theta_{2}\right)-T_{f 2}-k_{3}\left(\theta_{2}-\theta_{3}\right) \\
\vdots \\
J_{n-1} \ddot{\theta}_{n-1}=k_{n-1}\left(\theta_{n-2}-\theta_{n-1}\right)-T_{f n-1} \\
\quad-k_{n}\left(\theta_{n-1}-\theta_{n}\right) \\
J_{n} \ddot{\theta}_{n}=k_{n}\left(\theta_{n-1}-\theta_{n}\right)-T_{f n}
\end{array}\right.
$$

\section{B. The basic galvanometer modeling}

In case of the mirror single load $(n=1$ in (1)) as for the $X$ and $Y$ axis galvanometers, the Fig. 2 representation is simplified as in Fig. 3 where the $m$ index is used for the mirror parameters.

Equations (1) are simplified as in (2)

$$
\left\{\begin{array}{l}
U=R i+L \frac{d i}{d t}+E \\
J_{r} \ddot{\theta}_{r}=T_{r}-T_{f r}-k\left(\theta_{r}-\theta_{m}\right) \\
J_{m} \ddot{\theta}_{m}=k\left(\theta_{r}-\theta_{m}\right)-T_{f m}
\end{array}\right.
$$

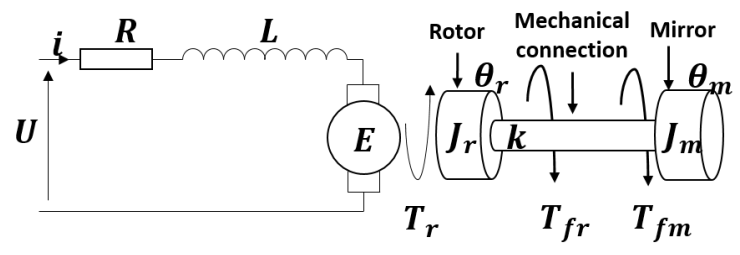

Fig. 3. Single mirror load galvanometer scanner

Standard simplified galvanometer modeling as in [3], [12] considers a rigid connection $\left(k \rightarrow \infty\right.$, yielding $\left.\theta_{r}=\theta_{m}\right)$ and negligible friction effects (air friction) at the mirror side $\left(T_{f m}=0\right)$. Considering only the viscous friction component on the rotor side, $T_{f r}$ is such as $T_{f r}=B_{r} \omega_{r}$, where $B_{r}$ is the viscous friction coefficient at the rotor side and $\omega_{r}=\frac{d \theta_{r}}{d t}$ is the angular velocity of the rotor. $E$ and $T_{r}$ are such that $E=K_{T} \omega_{r}$ and $T_{r}=K_{T} i, K_{T}$ being the value for both the torque constant and the counter-electromotive force constant of the motor. Equations (2) are simplified to

$$
\left\{\begin{array}{l}
U=R i+L \frac{d i}{d t}+K_{T} \frac{d \theta_{r}}{d t} \\
J_{r} \ddot{\theta}_{r}=K_{T} i-B \frac{d \theta_{r}}{d t}
\end{array}\right.
$$

yielding, with $s$ the Laplace variable, the commonly known simplified motor transfer function derived from (3) as:

$$
\frac{\theta_{r}(s)}{U(s)}=\frac{1}{s} \cdot \frac{K_{T}}{K_{T}^{2}+(s L+R)\left(s J_{r}+B_{r}\right)}
$$

hence the basic block diagram model of a galvanometer scanner depicted in Fig. 4.

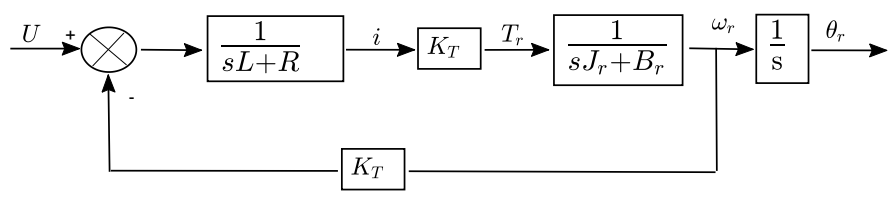

Fig. 4. Basic modeling of the galvanometer

Experimentations on a commercial three-axis scan head have been conducted in order to estimate the frequency responses of its galvanometers. The experimental frequency response is compared to the basic model frequency response on Fig. 5 for the $X$ axis galvanometer where it is observed

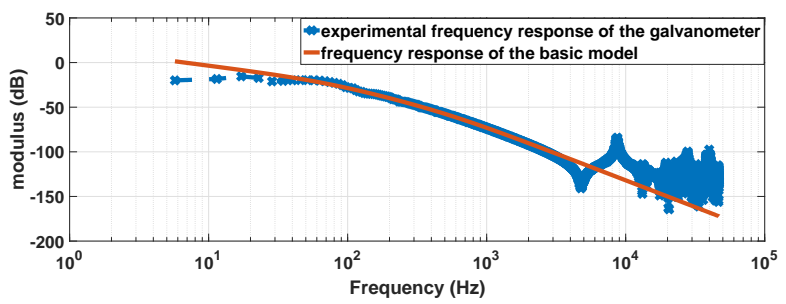

Fig. 5. Experimental and basic model frequency responses of the $X$ axis galvanometer

that the basic model shows disparities at low and high frequencies. These disparities are addressed in the proposed enhanced modeling. 


\section{Proposed enhanced galvanometer modeling}

1) Flexible modes modeling: The high frequencies phenomenon observed in Fig. 5 is due to fact that $k$ in Fig. 3 is actually finite, which yields a flexible resonance mode for the mechanical link. In general terms, finite $k_{j, j=1}$ to $n$ from the multi-loads representation in Fig. 2 yields $n$ flexible resonance modes. With finite $k_{j, j=1}$ to $n$ factors and considering viscous frictions at each small load stage as $T_{f j}=B_{j} \omega_{j}$, for $j=1$ to $n$, (1) becomes

$$
\left\{\begin{array}{l}
U=R i+L \frac{d i}{d t}+K_{T} \frac{d \theta_{r}}{d t} \\
J_{r} \ddot{\theta}_{r}=K_{T} i-B_{r} \frac{d \theta_{r}}{d t}-k_{1}\left(\theta_{r}-\theta_{1}\right) \\
J_{1} \ddot{\theta}_{1}=k_{1}\left(\theta_{r}-\theta_{1}\right)-B_{1} \frac{d \theta_{1}}{d t}-k_{2}\left(\theta_{1}-\theta_{2}\right) \\
J_{2} \ddot{\theta}_{2}=k_{2}\left(\theta_{1}-\theta_{2}\right)-B_{2} \frac{d \theta_{2}}{d t}-k_{3}\left(\theta_{2}-\theta_{3}\right) \\
\vdots \\
J_{n-1} \ddot{\theta}_{n-1}=k_{n-1}\left(\theta_{n-2}-\theta_{n-1}\right)-B_{n-1} \frac{d \theta_{n-1}}{d t} \\
\quad-k_{n}\left(\theta_{n-1}-\theta_{n}\right) \\
J_{n} \ddot{\theta}_{n}=k_{n}\left(\theta_{n-1}-\theta_{n}\right)-B_{n} \frac{d \theta_{n}}{d t}
\end{array}\right.
$$

To introduce the flexible modes, let us consider the current $i$ to velocity $\omega_{r}$ transfer function of the basic model of Fig. 4

$$
\left[\frac{\omega_{r}(s)}{i(s)}\right]_{\mathrm{basic}}=\frac{K_{T}}{s J_{r}+B_{r}}
$$

and compute from (5) the same transfer function $\left[\frac{\omega_{r}(s)}{i(s)}\right]_{\text {full }}$ with flexible modes. The electrical equation which is the first line of (5) will be omitted in the following calculations since it is not affected by the resonance phenomenon. By expressing the desired $\left[\frac{\omega_{r}(s)}{i(s)}\right]_{\text {full }}$ transfer function from the second line of (5) and computing for the remaining lines $\frac{\theta_{j}}{\theta_{j-1}}$, for $j=1$ to $n$ with $\theta_{0}=\theta_{r}$, we get:

$$
\left\{\begin{array}{l}
\frac{\omega_{r}(s)}{i(s)}=\frac{K_{T}}{s J_{r}+B_{r}+\frac{k_{1}}{s}\left(1-\frac{\theta_{1}(s)}{\theta_{r}(s)}\right)} \\
\frac{\theta_{1}(s)}{\theta_{r}(s)}=\frac{k_{1}}{s^{2} J_{1}+s B_{1}+k_{1}+k_{2}\left(1-\frac{\theta_{2}(s)}{\theta_{1}(s)}\right)} \\
\frac{\theta_{2}(s)}{\theta_{1}(s)}=\frac{k_{2}}{s^{2} J_{2}+s B_{2}+k_{2}+k_{3}\left(1-\frac{\theta_{3}(s)}{\theta_{2}(s)}\right)} \\
\vdots \\
\frac{\theta_{n-1}(s)}{\theta_{n-2}(s)}=\frac{k_{n-1}}{s^{2} J_{n-1}+s B_{n-1}+k_{n-1}+k_{n}\left(1-\frac{\theta_{n}(s)}{\theta_{n-1}(s)}\right)} \\
\frac{\theta_{n}(s)}{\theta_{n-1}(s)}=\frac{k_{n}}{s^{2} J_{n}+s B_{n}+k_{n}}
\end{array}\right.
$$

Let us define the operator degrees $(t f(s))=\frac{a}{b}$ such that $t f(s)$ is a rational transfer function, $a$ is the degree of the numerator of $t f(s)$ and $b$ is the degree of the denominator of $t f(s)$.

Starting from the last line of (7) and upwards, we get successively:

$$
\begin{aligned}
& \text { - degrees }\left(\frac{\theta_{n}}{\theta_{n-1}}\right)=\frac{0}{2} \\
& \text { - degrees }\left(\frac{\theta_{n-1}}{\theta_{n-2}}\right)=\frac{2}{4}
\end{aligned}
$$$$
\text { - : }
$$

- degrees $\left(\frac{\theta_{2}}{\theta_{1}}\right)=\frac{2(n-2)}{2(n-1)}$
- degrees $\left(\frac{\theta_{1}}{\theta_{n}}\right)=\frac{2(n-1)}{2 n}$

Moreover, a $s$ multiplicator can be factorized from the quantity $1-\frac{\theta_{n}(s)}{\theta_{n-1}(s)}$, then $1-\frac{\theta_{n-1}(s)}{\theta_{n-2}(s)}$ and so forth until $1-\frac{\theta_{1}(s)}{\theta_{r}(s)}$. In the end it gives

$$
\text { degrees }\left(\left[\frac{\omega_{r}(s)}{i(s)}\right]_{\text {full }}\right)=\frac{2 n}{2 n+1}
$$

Since degrees $\left(\left[\frac{\omega_{r}(s)}{i(s)}\right]_{\text {basic }}\right)=\frac{0}{1},\left[\frac{\omega_{r}(s)}{i(s)}\right]_{\text {full }}$ can be decomposed as follows

$$
\left[\frac{\omega_{r}(s)}{i(s)}\right]_{\text {full }}=\left[\frac{\omega_{r}(s)}{i(s)}\right]_{\text {basic }}+\left[\frac{\omega_{r}(s)}{i(s)}\right]_{\text {resonances }}
$$

with the condition: degrees $\left(\left[\frac{\omega_{r}(s)}{i(s)}\right]_{\text {resonances }}\right)=\frac{x}{y}$ such that $x \leq 2 n-1$ and $y=2 n$. The proposed flexible modes model is as:

$$
\left[\frac{\omega_{r}(s)}{i(s)}\right]_{\text {resonances }}=\sum_{j=1}^{n} \frac{K_{r j} s}{s^{2}+2 \xi_{r j} \omega_{r e s j} s+\omega_{r e s j}^{2}}
$$

with three adjustment parameters for each flexible resonance mode: a gain $K_{r j}$, a damping coefficient $\xi_{r j}$ and a resonance frequency $\omega_{\text {res } j}$.

For illustrative purpose, for the one mirror load case $(n=1$ and $\theta_{1}=\theta_{m}$ ), from (2), we compute:

$$
\left[\frac{\omega_{r}(s)}{i(s)}\right]_{\text {full }}=\frac{K_{T}\left(s^{2} J_{m}+s B_{m}+k\right)}{\left(s^{2} J_{m}+s B_{m}+k\right)\left(s J_{r}+B_{r}\right)+k\left(s J_{m}+k B_{m}\right)}
$$

which can be rewritten with the proposed modeling:

$$
\left[\frac{\omega_{r}(s)}{i(s)}\right]_{\text {full }}=\frac{K_{T}}{s J_{r}+B_{r}}+\frac{K_{r} s}{s^{2}+2 \xi_{r} \omega_{r e s} s+\omega_{r e s}^{2}}
$$

making (11) and (12) equivalent.

2) Motor friction modeling: To address the gain attenuation phenomenon at low frequencies observed in Fig. 5, friction studies are introduced. Experiments at different constant angular velocities values are performed, indeed motor torque $T_{r}$ and friction torque $T_{f r}$ are equal when angular velocity is constant and friction torque can be computed from current $i$ records. Comparison of the $X$ axis galvanometer friction records to the friction curve of the basic model of Fig. 4 is depicted in Fig. 6 where it is observed that the

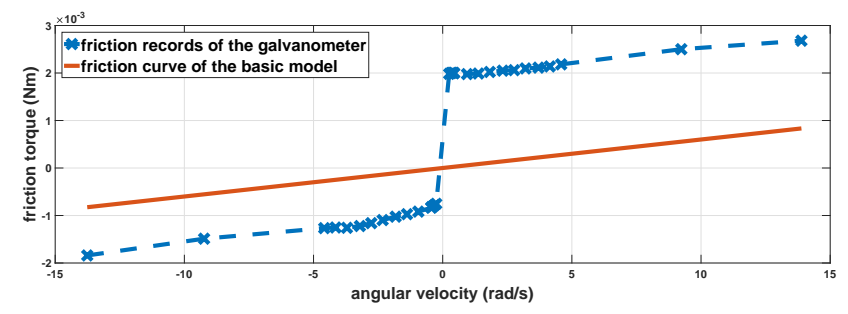

Fig. 6. Friction records of the galvanometer and the basic model

basic friction model $T_{f r}\left(\omega_{r}\right)=B_{r} \omega_{r}$ presents poor accuracy compared to real friction values of the galvanometer. A new 
friction model inspired by the modeling of DC motor various types of friction investigated in [14] is proposed. The retained model is as

$$
T_{f r}\left(\omega_{r}\right)=B_{r} \omega_{r}+T_{C} \operatorname{sign}\left(\omega_{r}\right)+T_{P},
$$

where $T_{C}$ is the Coulomb friction coefficient, a common friction element to take into account for two-way rotating motors. $T_{P}$ is the friction torque at the motor initial position (it reflects the mirror weight) since the initial zero angular position for the galvanometer is a non-zero current controlled position.

3) Summary of the proposed enhanced galvanometer modeling: The complete proposed flexible modes and friction enhanced model is depicted in Fig. 7.

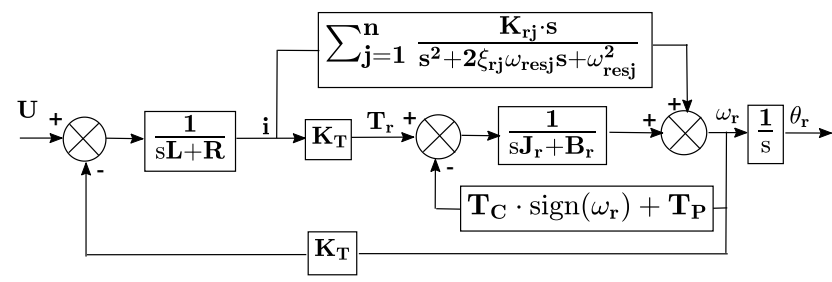

Fig. 7. Summary of the enhanced galvanometer model

\section{Identification of the parameters of the proposed gal- vanometer model}

The flexible modes and the friction model parameters are determined using experimental data from a commercial device, meaning however that the galvanometer is inserted in a controlled loop and cannot be disconnected. This entails open-loop model parameters identification from closedloop acquired data, mainly voltage signals $U$ and angular responses $\theta_{r}$. The angular reference signals used are variable magnitude velocity-limited step signal as depicted in Fig. 8. Such kind of signal is considered because it spans the whole

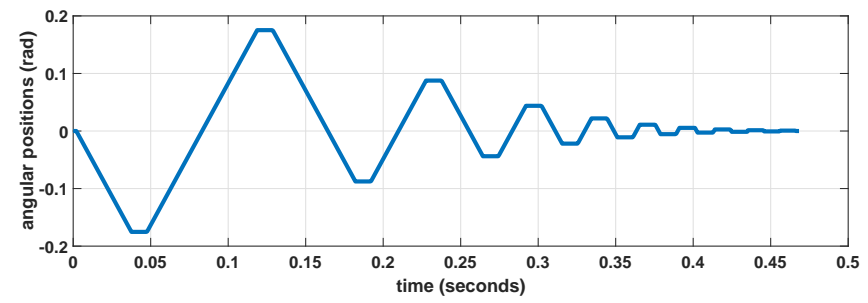

Fig. 8. The velocity-limited multi-step angular reference

angular values capabilities of the galvanometer actuator.

For the $X$ and $Y$ galvanometers, the flexible mode model order is set to $n=1$ since the considered motor load is just the deflecting mirror. For the $Z$ galvanometer, the parameter is set to $n=2$ given the more complex rotation to translation mechanism, this means that two frequency resonances are modeled for the $Z$-axis. The flexible modes parameters are tuned by hand accordingly to the experimental galvanometer frequency responses as depicted in Fig 5 for $X$ axis.
The friction model parameters are tuned using a Particle Swarm Optimisation (PSO) algorithm described in [16] as the 'perturbed' algorithm. PSO is an optimization method that uses random processes which yields no specific requirements on the problem. In this case the cost function structure (in particular the discontinuity of the 'sign' function in (13)) is not a problem since the method does not rely on gradient computation. Moreover, local minimas can be avoided with the random feature of PSO. The cost function to be minimized here is the Mean Square Error (MSE) between the experimental time response of the galvanometer and the time response of the model to the experimental voltage $U$ input signal.

Results of the frequency responses of the $X$ axis ( $Y$ axis results are similar to $X$ axis ones) and the $Z$ axis are presented in Fig. 9 and 10 where experimental frequency responses are compared to both the basic and the enhanced models. Results on the friction of the $X$ axis is presented in Fig. 11 (the shape of $Y$ and $Z$ axis friction curves are similar to the $X$ axis one) where experimental data are also compared to the basic and the enhanced model friction curves.

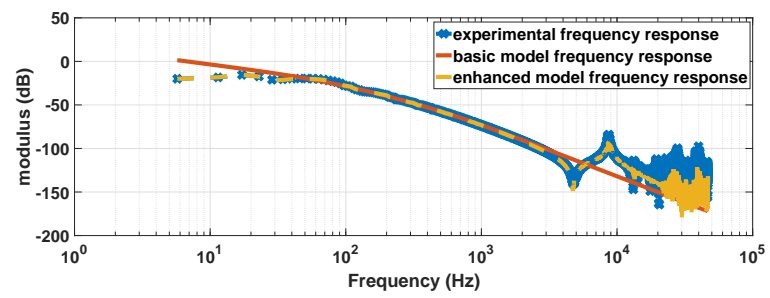

Fig. 9. $\quad X$ axis frequency responses

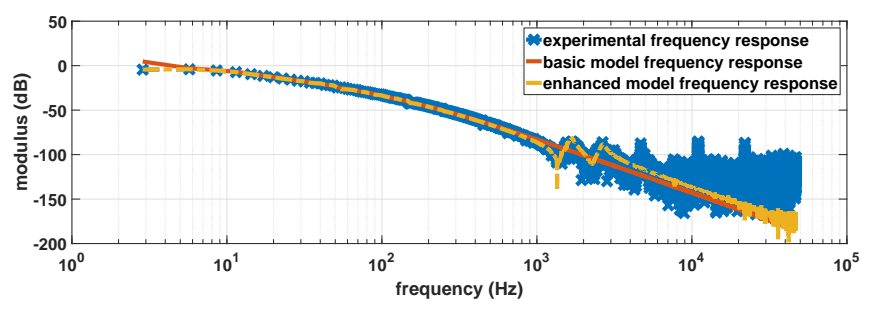

Fig. 10. $Z$ axis frequency responses

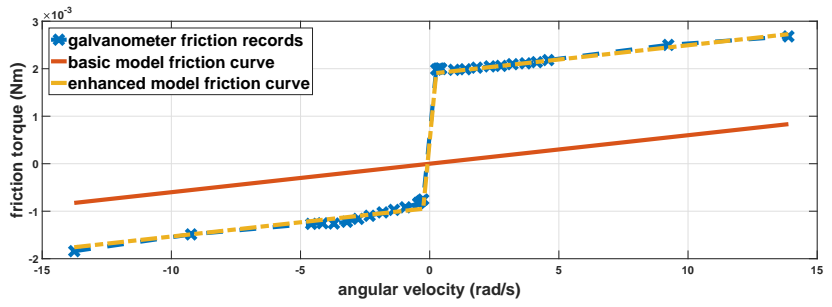

Fig. 11. $\quad X$ axis friction curves

It can be observed that the low and high frequencies behavior of the experimental frequency responses are correctly represented by the proposed enhanced model. The friction curves are also more representative of the experimental values. 


\section{EXPERIMENTAL VALIDATION OF THE MODELS}

In order to validate the developed galvanometer models of each axis, simulations on a simple job-marking shape in the $(x, y)$ working plane are launched. The marked shape, a simple square shape reference is depicted in Fig. 12. The

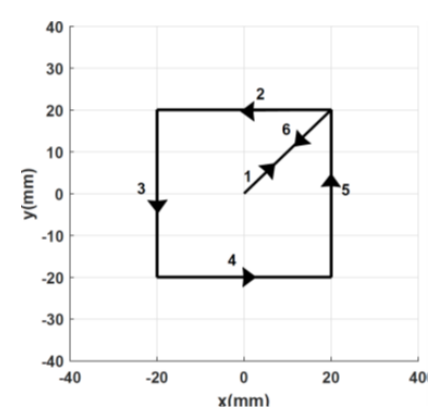

Fig. 12. Marking of a simple square shape

marking path starts and ends both at the $(x, y)=(0,0)$ position. The focusing plane height reference is constant to zero $z_{\text {ref }}=0 \mathrm{~mm}$, i.e. marking plane and focusing plane are to be mixed at all time. The square shape is programmed by means of $x, y$ and $z$ values in an user interface of the three axis scanhead. Recorded experimental data are the angular references $\theta_{x r}, \theta_{y r}$ and $\theta_{z r}$ sent by the commercial proprietary controller card to the three axis scanhead and the angular responses of each galvanometer $\theta_{x}, \theta_{y}$ and $\theta_{z}$. In order to simulate the galvanometer models of each axis, a stabilizing controller with performances similar to the commercial machine controller is needed since no a priori knowledge on the experimental set-up is available. Moreover geometrical models that transform angular values $\theta_{x}, \theta_{y}, \theta_{z}$ to cartesian space values $x, y, z$ are needed in order to evaluate the performance of the models in the marking plane.

\section{A. Stabilizing controllers for the galvanometer models}

Relevant control methods for galvanometer based systems have been thoroughly studied in [10] in which four points of controller synthesis are emphasized: feedback control, feedforward control, enhanced modelisation/identification methods and optimization/autotuning of the controller. The selected structure to reproduce the experimental set-up scan head behavior is a common control method for DC motors detailed in [8] for which the controller architecture consists of:

- a feedback controller as a three-loop cascade structure

- a filtered Proportional Derivative controller for the motor position : $\operatorname{PD}_{\theta}(s)=K_{\theta} \frac{1+T_{d \theta} s}{1+0.1 \cdot T_{d \theta} s}$

- a Proportional Integral controller for the motor velocity : $\mathrm{PI}_{\omega}(s)=K_{\omega}\left(1+\frac{1}{T_{i \omega} s}\right)$

- a Proportional Integral controller for the motor current : $\mathrm{PI}_{\mathrm{i}}(s)=K_{i}\left(1+\frac{1}{T_{i i} s}\right)$

- a feedforward controller for the angular velocity and the current with adjustment gains $K_{1}$ and $K_{2} \in[0,1]$ for the tuning of the anticipation actions to be in $[0 \%, 100 \%]$ as in [8].
The parameters of the whole controller structure are derived once again from a Particle Swarm Optimisation (PSO) algorithm with the implementation as in [16]. The optimal controller parameters values are also obtained by minimizing the MSE between the experimental time response of the galvanometer and the time response of the closed-loop structure model to the same variable magnitude velocitylimited step signal as depicted in Fig. 8. For illustration purpose, modeling errors on the angular position responses between the experimental data and the closed-loop model of the $Z$ axis are depicted in Fig. 13 where the maximum error

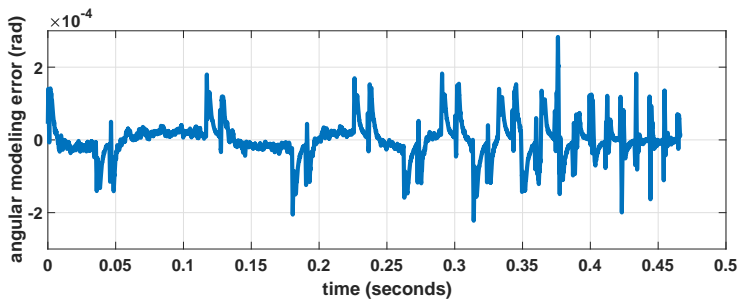

Fig. 13. Angular position responses difference between experimental data and the closed-loop model response for $Z$ axis

value is $2.83 \cdot 10^{-4} \mathrm{rad}$. For $X$ and $Y$ axis, the corresponding maximum modeling error values are $8.99 \cdot 10^{-5} \mathrm{rad}$ and $6.02 \cdot 10^{-5}$ rad. The angular modeling errors remain of an acceptable order of magnitude.

\section{B. Geometrical models for $\theta_{x}, \theta_{y}, \theta_{z}$ to $x, y, z$ transformation}

Geometrical transformation model is used to deduce marking plan coordinates from the angular values of the galvanometers axis. Many models exist depending on the accepted simplification hypotheses. For example, simplest geometrical models as in [4] considers $X$ and $Y$ galvanometers axis to be perfectly orthogonal. More restrictive hypotheses are often introduced to improve the geometrical modeling as in [15] where the previous hypothesis is eliminated. The geometrical model used here to illustrate the $x, y$ and $z$ axis performance is derived from [7] where the developed model takes into consideration a number of geometrical assembling defects. The description of this model, experimentally validated, related to the same scan head we consider in this paper, is not reproduced here due to place limitation.

\section{Experimental and simulation results comparison}

Now using the selected geometrical model, $(x, y)$ values from experimental data are compared to values obtained by simulation of the galvanometers models and their stabilizing controllers in Fig. 14 where corners and straight lines marking sections are zoomed in. The maximum contour error realized between experimental data and the model response is $153 \mu \mathrm{m}$ which is a relatively small value (for comparison typical laser beam waist is around the same order of magnitude).

Response of the $z$ height is depicted in Fig. 15 where it is observed that the model replicates well the behavior of the values computed from experimental data since the maximum $z$ value error between the two responses is equal to $16 \mu \mathrm{m}$. 

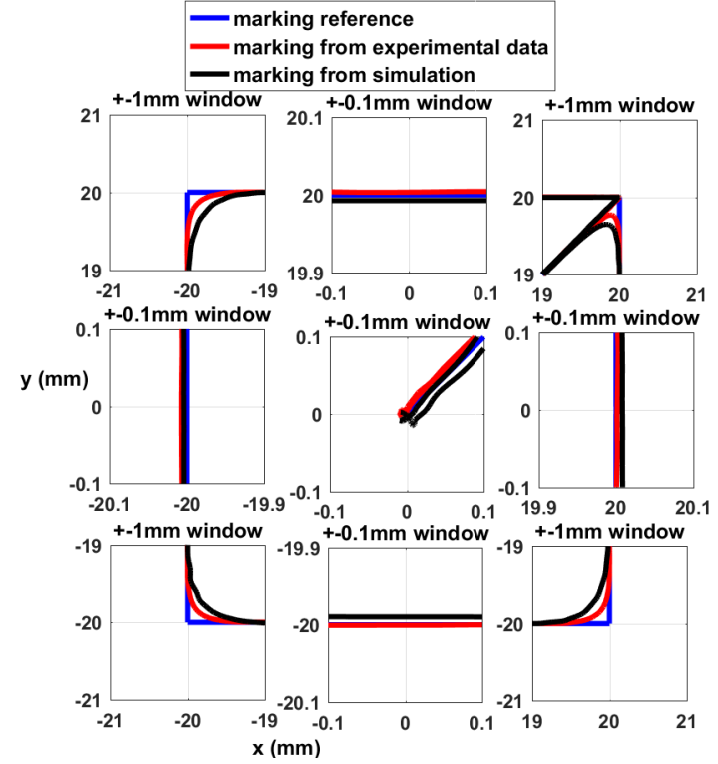

Fig. 14. $(x, y)$ marking plane comparison

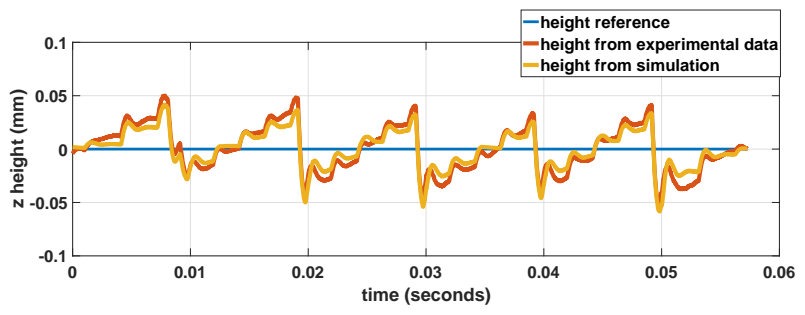

Fig. 15. $z$ heights comparison

As for maximum angular error between the experimental data and the model responses, the values for $\theta_{x}, \theta_{y}$ and $\theta_{z}$ are respectively $9.2 \cdot 10^{-5}, 8.5 \cdot 10^{-5}$ and $2.75 \cdot 10^{-5} \mathrm{rad}$. The angular modeling error values remain in a relatively small order of magnitude.

\section{CONCLUSION}

This paper establishes a new modeling approach for the galvanometer motor which is the mostly used actuator in the laser marking systems for the SLM process. The proposed model is enhanced with torsional flexible modes and physical friction consideration. The main advantage of the model is the extension of its frequency domain validation. Yet, depending on the model accuracy requirement, it can be improved with higher resonance mode order and advanced friction model functions as studied in [14]. The model performance presents admissible error values compared to experimental data in terms of both frequency and time responses of the motor. At this stage, a comprehensive three axis virtual environment is therefore available, realistic of the real behavior of a commercial scan head, which can be further used for performance improvement of the SLM process. The simulation approach used in this paper, paired with the laser beam parameters, can allow an upstream study of the marking process quality by means of actuator simulation. Furthermore, the proposed galvanometer motor model will be the entry point for advanced control structures investigation aiming to improve axis dynamics.

\section{ACKNOWLEDGMENTS}

This work takes part in the SOFIA project context. SOFIA is an applied research program for metal additive manufacturing, initiated by the Fives Michelin Additive Solutions jointventure (AddUP brand). SOFIA is funded by the Auvergne Rhône-Alpes region as well as Bpifrance as a structuring $\mathrm{R} \& \mathrm{D}$ project for competitiveness, within the 'Investing for the Future' program.

\section{REFERENCES}

[1] Aylward, R. P. (2003). Advanced galvanometer-based optical scanner design. Sensor review, 23(3), 216-222.

[2] Buchbinder, D., Schleifenbaum, H. B., Heidrich, S., Meiners, W., \& Bltmann, J. (2011). High power selective laser melting (HP SLM) of aluminum parts. Physics Procedia, 12, 271-278.

[3] Chen, C. T., Cheng, M. Y., Wu, C. H., \& Wen, C. M. (2011, May). Taguchi method based model predictive control design for laser scanner. In Control Conference (ASCC), 2011 8th Asian (pp. 737-742). IEEE.

[4] Duma, V. F. (2009). Mathematical functions of a 2-D scanner with oscillating elements. In Modeling, Simulation and Control of Nonlinear Engineering Dynamical Systems (pp. 243-253). Springer, Dordrecht.

[5] Feng, J., \& Zhi, J. (2011, August). Digital implementation of a galvanometric optical scanner based on DSP and FPGA. In Mechatronic Science, Electric Engineering and Computer (MEC), 2011 International Conference on (pp. 1899-1902). IEEE.

[6] Gebhardt, A. (2012). Understanding additive manufacturing: rapid prototyping-rapid tooling-rapid manufacturing. Carl Hanser Verlag $\mathrm{GmbH}$ Co KG.

[7] Godineau, K., Lavernhe, S. \& Tournier, C. (2017, October). Optomechanical modelling of an additive manufacturing laser scanning head including assembly defects. 4th joint Special Interest Group meeting between EUSPEN and ASPE on Dimensional Accuracy and Surface Finish from Additive Manufacturing, 53-57

[8] Godoy, E. (2007). Regulation industrielle. Paris: Dunod.

[9] Herzog, D., Seyda, V., Wycisk, E., \& Emmelmann, C. (2016). Additive manufacturing of metals. Acta Materialia, 117, 371-392.

[10] Iwasaki, M., Seki, K., \& Maeda, Y. (2012). High-precision motion control techniques: A promising approach to improving motion performance. IEEE Industrial Electronics Magazine, 6(1), 32-40.

[11] Mirtchev, T., Weeks, R., \& Minko, S. (2010, September). Optimizing the feedback control of Galvo scanners for laser manufacturing systems. In Photonics North 2010 (Vol. 7750, p. 77500T). International Society for Optics and Photonics.

[12] Mnerie, C. A., Preitl, S., \& Duma, V. F. (2015, May). Classical PID versus predictive control solutions for a galvanometer-based scanner. In Applied Computational Intelligence and Informatics (SACI), 2015 IEEE 10th Jubilee International Symposium on (pp. 349-353). IEEE.

[13] Murr, L. E., Gaytan, S. M., Ramirez, D. A., Martinez, E., Hernandez, J., Amato, K. N., ... \& Wicker, R. B. (2012). Metal fabrication by additive manufacturing using laser and electron beam melting technologies. Journal of Materials Science \& Technology, 28(1), 114.

[14] Olsson, H., Astrom, K. J., De Wit, C. C., Gfvert, M., \& Lischinsky, P. (1998). Friction models and friction compensation. Eur. J. Control, 4(3), 176-195.

[15] Xie, J., Huang, S., Duan, Z., Shi, Y., \& Wen, S. (2005). Correction of the image distortion for laser galvanometric scanning system. Optics \& Laser Technology, 37(4), 305-311.

[16] Yagoubi, M., \& Sandou, G. (2011). Particle Swarm Optimization for the design of $\mathrm{H} \infty$ static output feedbacks. IFAC Proceedings Volumes, 44(1), 12581-12586.

[17] Zhiqiang, D., Zude, Z., Wu, A., \& Youping, C. (2007). A linear drive system for the dynamic focus module of SLS machines. The International Journal of Advanced Manufacturing Technology, 32(1112), 1211-1217. 